\title{
Measuring Nearby Star Forming Regions with the VLBA
}

\author{
Rosa M. Torres ${ }^{* \dagger}$ \\ University of Bonn \\ E-mail: rtorreseastro.uni-bonn.de
}

This work is part of a very large ongoing effort to determine the distance and structure of all starforming regions within several hundred parsecs of the Sun using multi-epoch Very Long Baseline Interferometric observations. We will present the main results of Taurus and Ophiuchus (the two nearest low-mass star-forming regions): (1) the distance with accuracies of 0.5 to $6 \%$; (2) the impact and consequences of accurate measurements to our understanding of the structure and dynamics of those regions.

ISKAF2010 Science Meeting

June 10 -14 2010

Assen, the Netherlands

\footnotetext{
* Speaker.

${ }^{\dagger}$ Acknowledges support by the Deutsche Forschungsgemeinschaft (DFG) through the Emmy Noether Research grant VL 61/3-1.
} 


\section{Introduction}

An accurate trigonometric parallax measurement is a pre-requisite to the derivation, from observational data, of the most important characteristics (luminosity, age, mass, etc.) of a star. Unfortunately, even in the current post-Hipparcos era, the distance to young stars is rarely known to better than 20 to $30 \%$ (e.g. [1], [2]). At this level of accuracy, the luminosity of any given star cannot be assessed to better than 50\%. As a consequence, the accuracy with which young stars can be positioned on an H-R diagram is rather limited, and the comparison between observations and detailed theoretical models can only be approximate. This unsatisfactory state of affairs is largely the result of the fact that young stars are still embedded in their opaque parental cloud. They are, therefore, dim in the visible bands that were observed by Hipparcos.

Much of what we know about the formation of stars has been derived from the observation and modeling of a few nearby regions (Taurus, Ophiuchus, Perseus, Orion, Serpens). Thus, a significant improvement in the determination of the distance to these few regions would represent a major step forward. We will concentrate here on the two nearest regions of low-mass star formation: Taurus and Ophiuchus.

Since optical and near-infrared observations are affected by dust obscuration, one must turn to longer wavelengths to make progress in the determination of the parallax of young stars. Currently, the astrometry quality provided by mid- and far-infrared data as well as by sub-millimeter observations remains poor. The radio domain (particularly at $1 \mathrm{~cm} \lesssim \lambda \lesssim 10 \mathrm{~cm}$ ) provides, by far, the best prospect because large radio-interferometers can deliver extremely accurate astrometry (better than a tenth of a milli-arcsecond).

\section{Observations with VLBA}

Our sample contains a total of seven young stellar objects: five in the Taurus complex (T Tau Sb, HDE 283572, Hubble 4, HP Tau/G2, and V773 Tau A), and two in the Ophiuchus region (S1 and DoAr 21). All 7 objects were previously known to be fairly bright non-thermal radio sources, detectable using Very Long Baseline Interferometers. For each source, we obtained a series of $3.6 \mathrm{~cm}(8.42 \mathrm{GHz})$ continuum observations using the Very Long Baseline Array (VLBA) of the National Radio Astronomy Observatory (NRAO). The number of observations in each series as well as the cadence at which they were obtained were adjusted to each source. The details of the calibration are described further in [3]. The typical angular resolution of our final images was 1 to 2 milli-arcsecond, and the typical astrometric accuracy of each observation is 0.05 to 0.1 milliarcseconds for the sources in Taurus, and 0.2 to 0.6 milli-arcseconds in Ophiuchus. The poorer performance in Ophiuchus is related to the lower declination of this region $\left(\delta=-24^{\circ}\right.$ against $\delta=+20^{\circ}$ for Taurus).

\section{Proper motions, parallaxes and distances}

The displacement of a source on the celestial sphere is the combination of its trigonometric parallax $(\pi)$ and its proper motion $(\mu)$. In what follows, we will have to consider three different situations in terms of proper motions. 
Table 1: Astrometric parameters obtained for five stars in Taurus and two in Ophiuchus.

\begin{tabular}{|c|c|c|c|c|c|}
\hline Source & $\begin{array}{c}\mu_{\alpha} \cos \delta \\
{\left[\mathrm{mas} \mathrm{yr}^{-1}\right]}\end{array}$ & $\begin{array}{c}\mu_{\delta} \\
{\left[\mathrm{mas} \mathrm{yr}^{-1}\right]}\end{array}$ & $\begin{array}{c}\pi \\
{[\mathrm{mas}]}\end{array}$ & $\begin{array}{c}d \\
{[\mathrm{pc}]}\end{array}$ & $\begin{array}{l}\text { No. } \\
\text { Obs. }\end{array}$ \\
\hline $\mathrm{T}$ Tau Sb $*$ & $4.02 \pm 0.03$ & $-1.18 \pm 0.05$ & $6.82 \pm 0.03$ & $146.7 \pm 0.6$ & 12 \\
\hline Hubble 4 & $4.30 \pm 0.05$ & $-28.90 \pm 0.30$ & $7.53 \pm 0.03$ & $132.8 \pm 0.5$ & 6 \\
\hline HDE 2835724 & $8.88 \pm 0.06$ & $-26.60 \pm 0.10$ & $7.78 \pm 0.04$ & $128.5 \pm 0.6$ & 6 \\
\hline HP Tau/G2 & $13.85 \pm 0.03$ & $-15.40 \pm 0.20$ & $6.20 \pm 0.03$ & $161.2 \pm 0.9$ & 8 \\
\hline V773 Tau A .... & $17.27 \pm 0.14$ & $-23.79 \pm 0.09$ & $7.57 \pm 0.20$ & $130.0 \pm 2.0$ & 19 \\
\hline S1 & $-3.88 \pm 0.87$ & $-31.55 \pm 0.69$ & $8.55 \pm 0.50$ & $116.9 \pm 7.1$ & 6 \\
\hline DoAr 21 & $-26.47 \pm 0.92$ & $-28.23 \pm 0.73$ & $8.20 \pm 0.37$ & $121.9 \pm 5.5$ & 7 \\
\hline
\end{tabular}

$*$ The acelerations terms obtained for this fit were $a_{\alpha}=1.53 \pm 0.13 \mathrm{mas} \mathrm{yr}^{-2}$ in right

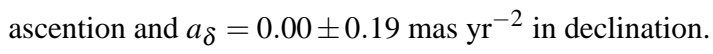

Single Stars. Two of our target stars are apparently single (HDE 283572 and Hubble 4), and one (HP Tau/G2) is a member of a multiple system with an orbital period so much longer than the timespan covered by the observations that the effect of the companions can safely be ignored. Thus, in these three cases, the proper motion can be assumed to be linear and uniform, and the right ascension $(\alpha)$ and the declination $(\delta)$ vary as a function of time $t$ as: $\alpha(t)=\alpha_{0}+\left(\mu_{\alpha} \cos \delta\right) t+\pi f_{\alpha}(t)$ and $\delta(t)=\delta_{0}+\mu_{\delta} t+\pi f_{\delta}(t)$, where $\alpha_{0}$ and $\delta_{0}$ are the coordinates of the source at a given reference epoch, $\mu_{\alpha}$ and $\mu_{\delta}$ are the components of the proper motion, and $f_{\alpha}$ and $f_{\delta}$ are the projections over $\alpha$ and $\delta$, respectively, of the parallactic ellipse (see also [4], [5] and [3]).

Binary Systems. One source (T Tau Sb) is a member of a binary system with an orbital period longer than the timespan of our observations but not by a huge factor. While a full Keplerian fit would again, in principle, be needed, we found that including a constant acceleration term provides an adequate description of the trajectory. The fitting functions in this case are of the form: $\alpha(t)=$ $\alpha_{0}+\left(\mu_{\alpha 0} \cos \delta\right) t+\frac{1}{2}\left(a_{\alpha} \cos \delta\right) t^{2}+\pi f_{\alpha}(t)$ and $\delta(t)=\delta_{0}+\mu_{\delta 0} t+\frac{1}{2} a_{\delta} t^{2}+\pi f_{\delta}(t)$, where $\mu_{\alpha 0}$ and $\mu_{\delta 0}$ are the proper motions at a reference epoch, and $a_{\alpha}$ and $a_{\delta}$ are the projections of the uniform acceleration (for details see [6]).

Tight Binaries. Three of our sources (V773 Tau A, S1 and DoAr 21) are compact binary systems with an orbital period of the order of the timespan covered by the observations. In such a situation, one should fit simultaneously for the uniform proper motion of the center of mass and for the Keplerian orbit of the system. This requires more observations than are needed to fit only for a uniform proper motion. Additional data were collected to adequately constrain the required fits but here we will present preliminary results based on equations for single stars where the Keplerian motion is not included. Note that the main effect of not fitting for the Keplerian orbit is an increase in the final uncertainty on the distance (see [7] and [3]).

The astrometric parameters are shown in Tab. 1 and were determined by least-squares fitting the data points with either equations using a Singular Value Decomposition (SVD) scheme. To check our results, we also performed two other fits to the data, a linear one based on the associated normal equations, and a non-linear one based on the Levenberg-Marquardt algorithm. They gave results identical to those obtained using the SVD method. The details of the astrometry fits are described 
further in [3].

\section{Three dimensional structure of Taurus and Ophiuchus}

The total spatial extent of Taurus on the sky is about $10^{\circ}$, corresponding to a physical size of about $25 \mathrm{pc}$ [5]. Our observations show that the depth of the complex is similar since HP Tau/G2 is about $30 \mathrm{pc}$ farther than Hubble 4, HDE 283572 or V773 Tau A. This has an important consequence: even if the mean distance of the Taurus association were known to infinite accuracy, one would still make errors as large as $10-20 \%$ by using the mean distance indiscriminately for all sources in Taurus. To reduce this systematic source of error, one needs to establish the three-dimensional structure of the Taurus association, and observations similar to those presented here currently represent the most promising avenue toward that goal. Indeed, the observations of the five stars presented here already provide some hints of what the three-dimensional structure of Taurus might be (see Fig. 1).

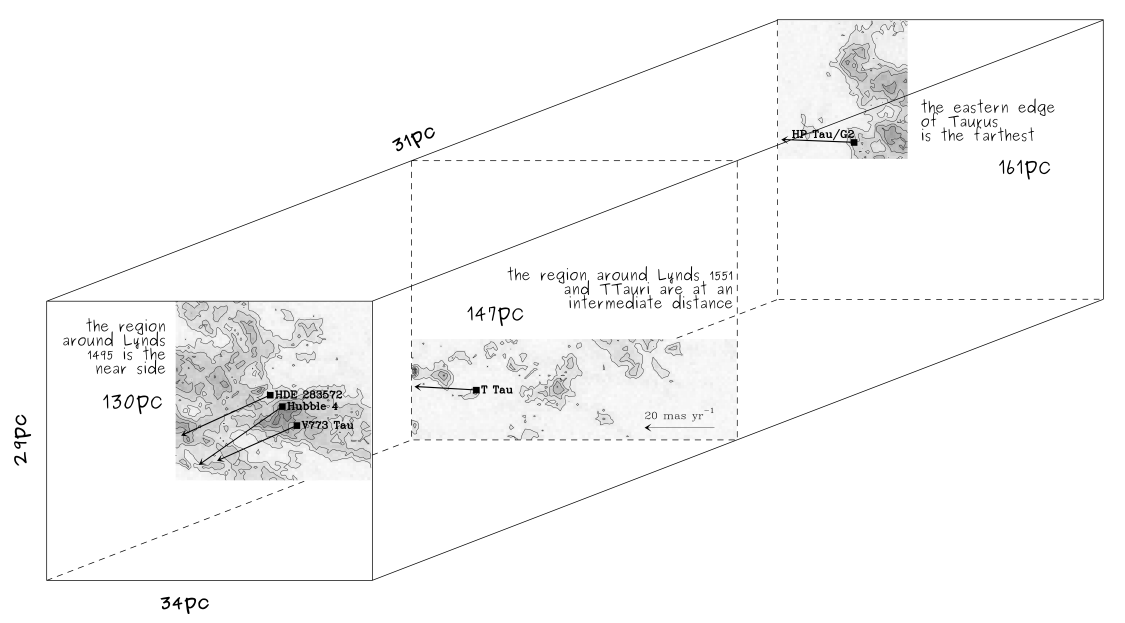

Figure 1: Sketch of the three-dimensional structure of Taurus made with a $\mathrm{CO}(1-0)$ map [8]. Five sources were superposed on the map, and the arrows are shown its tangential velocity. Hubble 4, HDE 283572, and V773 Tau A were found to be at about $130 \mathrm{pc}$, and are also located in the same portion of Taurus. T Tau Sb is located in the southern part of Taurus and it appears to be somewhat farther from us (note that its tangential velocity is clearly different from the other sources). HP Tau/G2 is located near the (Galactic) eastern edge of Taurus, and it is the farthest source.

Ophiuchus is composed of a compact core, only about $2 \mathrm{pc}$ across, and filamentary structures (called "streamers") extending (in projection) to about $10 \mathrm{pc}$ (see Fig. 2). The Ophiuchus core is sufficiently compact that we do not expect to resolve any structure along the line of sight, and our observations show that it is at a distance of $120 \mathrm{pc}$ [7]. There could potentially be distance gradients of several parsecs across the streamers. We note, however, that [9] deduced a distance of Haro 1-14C (associated with the darks clouds L1709/L1704) of $111 \pm 19 \mathrm{pc}$, suggering that the northern streamer is somewhat closer that the core. On the other hand, [10] determined a distance of $178_{-37}^{+18} \mathrm{pc}$ to the protostar IRAS 16293-2422 deeply embedded in L1689 (the southern Ophiuchus streamer), which would be more consistent with the older value of $165 \mathrm{pc}$. Even including the streamers, Ophiuchus is only $10 \mathrm{pc}$ across in projection, so it is unlikely to be $60 \mathrm{pc}$ deep. Thus, if the results of [10] are confirmed, they would indicate the existence of several unrelated star-forming regions along the line of sight. More observations -some of which have already been collected and partially analyzed- will be necessary to settle this issue. 


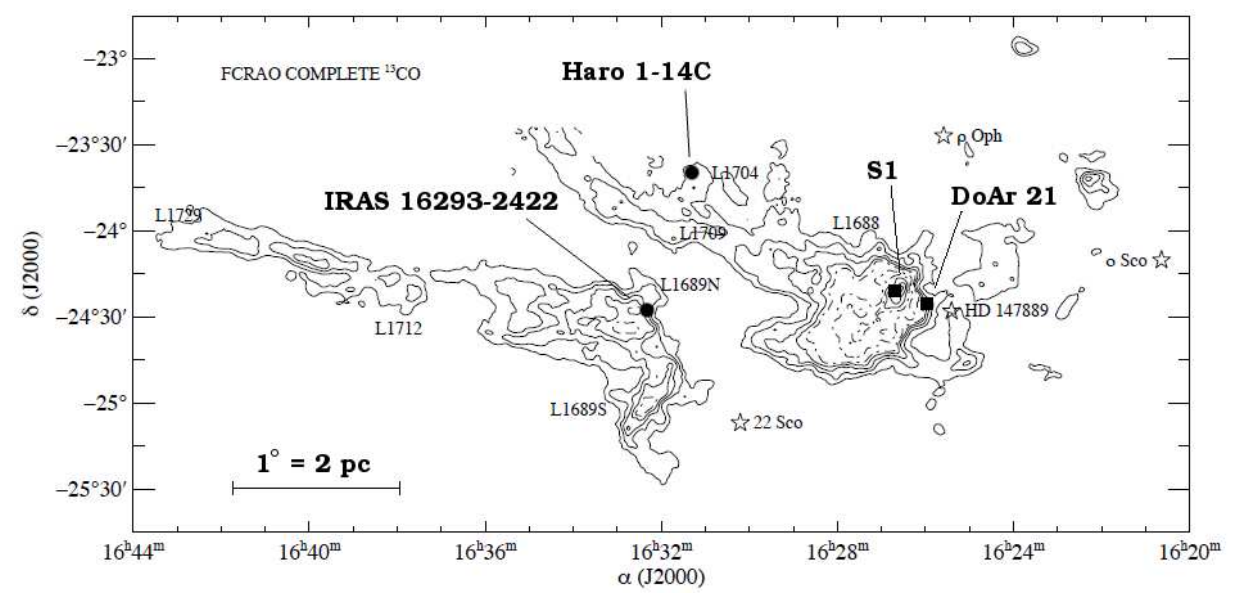

Figure 2: Positions of four stars superposed on the ${ }^{13} \mathrm{CO}$ map of Ophiuchus from [11]. The two stars studied here (S1 and DoAr 21) are shown as a squares; Haro 1-14C and IRAS 16293-2422 are shown as a circles.

\section{Implications for the properties of the stars}

Having obtained accurate distances to several young stars, we are now in a position to refine the determination of the intrinsic properties of each star. We will discuss here only the case of HP Tau/G2 and HDE 283572 (for a detailed discussion of other stars see [3]).

HP Tau/G2 is a member of a compact group of four young stars, comprising HP Tau itself, HP Tau/G1, HP Tau/G2, and HP Tau/G3 (hereafter HP, G1, G2 and G3 respectively). Given the small angular separations between them, the members of this group are very likely to be physically associated -indeed, G2 and G3 are thought to form a bound system. They are, therefore, very likely to be at the same distance from the Sun. Using our accurate estimate of the distance to G2, we are now in a position to refine the determination of the luminosities of all four stars. Little is known about G1, but the effective temperature and the bolometric luminosity (obtained assuming $d=142$ pc) of the other three members are given in [12]. Those values (corrected to the new distance) allow us to place the stars accurately on an HR diagram (Fig. 3).

From their position on the HR diagram, one can (at least in principle) derive the mass and age of the stars using theoretical pre-main sequence evolutionary codes. Several such models are available, and we will use four of them here: those of [13], [14], [15], and [16]. The isochrones for those four models at 1, 3, 5, 7, and $10 \mathrm{Myr}$ are shown as solid black lines in Fig. 3. Also shown are the evolutionary tracks (from the same models) for stars of 1.0, 1.5, and $2.0 \mathrm{M}_{\odot}$.

A number of interesting points can be seen from Fig. 3. First, there is reasonable agreement (within $40 \%$ ) between the masses predicted by different models. For G2 different models predict masses consistent with each other at the $10 \%$ level (between 1.7 and $1.9 \mathrm{M}_{\odot}$ ). For HP the models of [13] or [16] predict a mass of $\sim 1.5 \mathrm{M}_{\odot}$, whereas those of [15] predicts a significantly smaller mass of $\sim 1.0 \mathrm{M}_{\odot}$; there is a $35 \%$ spread in the values predicted by different models for the mass of this source. For G3 the mass is about $0.8 \mathrm{M}_{\odot}$ according to the models of [13], but slightly less than 0.5 $\mathrm{M}_{\odot}$ according to those of [15]; this is a $40 \%$ discrepancy.

Since the different members of the HP Tau group are likely to be physically associated, they are expected to be nearly coeval. Interestingly, most models predict significantly different ages for the 

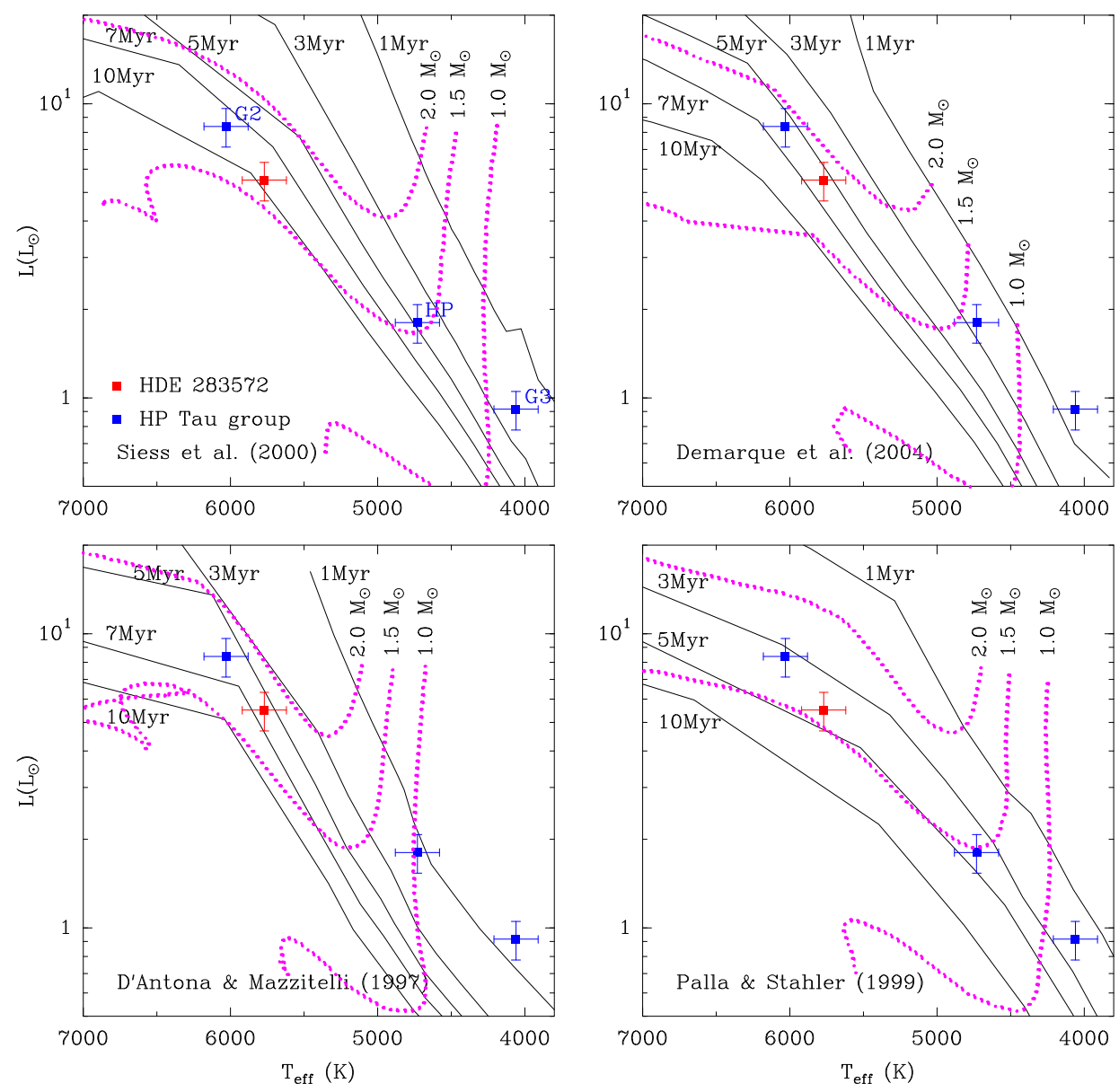

Figure 3: Positions of the three HP Tau members (blue symbols) and of HDE 283572 (red symbol) on an HR diagram. From the coolest to the warmest, the three stars in the HP Tau group are HP Tau/G3, HP Tau, and HP Tau/G2, as indicated in the first panel. Isochrones (full back lines) are shown at 1, 3, 5, 7, and $10 \mathrm{Myr}$ for various models. For the same models, evolutionary tracks for stars of 1.0, 1.5, and $2.0 \mathrm{M}_{\odot}$ are also shown as dotted magenta lines.

three sources (see Fig. 3). The models by [13] predict ages of about $8 \mathrm{Myr}$ and $3 \mathrm{Myr}$ for G2 and G3, respectively. A similar 5 Myr age difference is found for the models of [14] and [15]: both predict ages slightly smaller than $1 \mathrm{Myr}$ for G3, and somewhat larger than $5 \mathrm{Myr}$ for G2. It is possible that those differences could be real, however, it should be noticed that the vast majority of low-mass stars in Taurus (with spectral types M and late K) have ages smaller than 3 Myr [12]. Moreover, mass-dependent systematic effects in the age predictions made by evolutionary tracks have been reported before, and existing models could significantly over-predict the age of relatively massive stars $\left(\mathrm{M} \gtrsim 1.5 \mathrm{M}_{\odot}\right)$. $\mathrm{G} 2$ is precisely such a fairly massive star. So, the age estimate for HDE 283572 based on the models by [13], [14] and [15] is 6-10 Myr (Fig. 3), somewhat larger than would be expected for Taurus. The only of the four models considered here to predict similar ages for the three members of the HP Tau group is that of [16]. Within the errors, all three stars fall on the $3 \mathrm{Myr}$ isochrone. Note that this value is also consistent with the ages of lower mass stars in Taurus [12]. 


\section{References}

[1] Knude, J., \& Hog, E. Interstellar reddening from the HIPPARCOS and TYCHO catalogues. I. Distances to nearby molecular clouds and star forming regions, A\&A, 338 (1998) 897

[2] Bertout, C., Robichon, N., \& Arenou, F., Revisiting Hipparcos data for pre-main sequence stars, A\&A, 352 (1999) 574

[3] Torres, R. M., Measuring Nearby Star Forming Regions with the VLBA: from the Distance to the Dynamics, UNAM \& NRAO, PhD Thesis (2010)

[4] Torres, R. M., Loinard, L., Mioduszewski, A. J., \& Rodriguez, L. F., VLBA Determination of the Distance to Nearby Star-Forming Regions. II. Hubble 4 and HDE 283572 in Taurus, ApJ, 671 (2007) 1813

[5] Torres, R. M., Loinard, L., Mioduszewski, A. J., \& Rodriguez, L. F., VLBA Determination of the Distance to Nearby Star-Forming Regions. III. HP Tau/G2 and the 3D Structure of Taurus Cloud, ApJ, 698 (2009) 242

[6] Loinard, L., Torres, R. M., Mioduszewski, A. J., Rodríguez, L. F., González-Lópezlira, R. A., Lachaume, R., Vázquez, V., \& González, E., VLBA Determination of the Distance to Nearby Star-Forming Regions. I. The distance to T Tauri with $0.4 \%$ accuracy, ApJ, 671 (2007) 546

[7] Loinard, L., Torres, R. M., Mioduszewski, A. J., \& Rodríguez, L. F., A Preliminary Distance to the Core of Ophiuchus, with an Accuracy of 4\%, ApJ, 675 (2008) L29

[8] Dame, T. M., Hartmann, D., \& Thaddeus, P., The Milky Way in Molecular Clouds: A New Complete CO Survey, ApJ, 547 (2001) 792

[9] Schaefer, G. H., Simon, M., Prato, L., \& Barman, T., Preliminary Orbit of the Young Binary Haro $1-14 c$, AJ, 135 (2008) 1659

[10] Imai, H., Nakashima, K., Bushimata, T., Choi, Y. K., Hirota, T., Honma, M., Horiai, K., Inomata, N., Iwadate, K., Jike, T., Kameya, O., Kamohara, R., Kan-Ya, Y., Kawaguchi, N., Kijima, M., Kobayashi, H., Kuji, S., Kurayama, T., Manabe, S., Miyaji, T., Nagayama, T., Nakagawa, A., Oh, C. S., Omodaka, T., Oyama, T., Sakai, S., Sakakibara, S., Sato, K., Sasao, T., Shibata, K. M., Shimizu, R., Shintani, M., Sofue, Y., Sora, K., Suda, H., Tamura, Y., Tsushima, M., Ueno, Y., \& Yamashita, K., Astrometry of H2O Masers in Nearby Star-Forming Regions with VERA. I. IRAS 16293-2422 in $\rho$ Oph East, PASJ, 59 (2007) 1107

[11] Ridge, N. A., Di Francesco, J., Kirk, H., Li, D., Goodman, A. A., Alves, J. F., Arce, H. G., Borkin, M. A., Caselli, P., Foster, J. B., Heyer, M. H., Johnstone, D., Kosslyn, D. A., Lombardi, M., Pineda, J. E., Schnee, S. L., \& Tafalla, M., The COMPLETE Survey of Star-Forming Regions: Phase I Data, AJ, 131 (2006) 2921

[12] Briceño, C., Luhman, K. L., Hartmann, L., Stauffer, J. R., \& Kirkpatrick, J. D., The Initial Mass Function in the Taurus Star-forming Region, ApJ, 580 (2002) 317

[13] Siess, L., Dufour, E., \& Forestini, M., An internet server for pre-main sequence tracks of low-and intermediate-mass stars, A\&A, 358 (2000) 593

[14] Demarque, P., Woo, J.-H., Kim, Y.-C., \& Yi, S. K., Y2 Isochrones with an Improved Core Overshoot Treatment, ApJS, 155 (2004) 667

[15] D’Antona, F., \& Mazzitelli, I., Evolution of low mass stars, MmSAI, 68 (1997) 807

[16] Palla, F., \& Stahler, S. W., Star Formation in the Orion Nebula Cluster, ApJ, 525 (1999) 772 